\title{
W. A. Bassow - der Begründer der chirurgischen Magenoperationslehre. Werdegang der Magenchirurgie im 19. Jahrhundert
}

Wassili Alexandrowitsch Bassow (1812-1879) war Ordinarius an der Moskauer Universität. Er galt als der bekannteste und beste Chirurg und Arzt seiner Zeit, der vielfach unentgeltlich arbeitete. Nach Aussagen von L. F. Zmejew kam W. A. Bassow „einem Vorbild des Evangeliums nahe“. Als erster wandte er viele verbesserte Verfahren westlicher Wissenschaft in Russland an. Bereits als Prosektor führte er in Moskau Vivisektionenein und schon zu Beginn der 1850er Jahre die erste in Moskau vorgenommene Tracheotomiebei Kehlkopftuberkulose durch, bei dem Arzt Iwanow.

Der bekannte Ausspruch a Jove principium (,mit Jupiter anfangen“) bedeutet in der Lehre der Alten, die Betrachtung einer geschichtlichen Entwicklung mit der Entdeckung oder Erfindung, die als Startpunkt einer neuen wissenschaftlichen Strömung gilt, zu beginnen. Als eine solche Erfindung in der Geschichte der experimentellen Untersuchung der Magenfunktion und der Magenchirurgie kann der von Bassow 1842 entwickelte ,künstliche Weg in den Magen der Tiere“ gelten - die künstliche Magenfistel. Nach der berechtigten Einschätzung von Pawlowwurde diese Erfindung zu einem „Ausgangspunkt moderner Methodik“ bei der Erforschung der Magenfunktionen.

Wassili Alexandrowitsch Bassow, habilitierter Doktor der Medizin und Chirurg, wurde im Jahre 1812 geboren. 1833 absolvierte er das Studium an der Medizinischen Fakultät der Moskauer Universität; 1841 habilitierte er dort zum Doktor der Medizin mit der Schrift „Zur Steinkrankheit der Harnblase im allgemeinen und insbesondere zur Steinentfernung durch den Dammschnitt“. Im September 1842 legte er im Rahmen eines Hundeexperiments die weltweit erste künstliche Magenfistel in der Geschichte der Wissenschaft an. 1843 bis 1844 begab sich Bassow auf eine Dienstreise ins Ausland, nach deren Ende er zum Adjunkt und Lektor der theoretischen Chirurgie ernannt wurde. Im Jahr 1848 erschien in Sankt Petersburg sein Werk „Zur Bedeutung der Chirurgie im Kreise der Heilwissenschaften “. Ebenfalls seit 1848 wirkte er als außerordentlicher, seit 1852 als ordentlicher Professor der Moskauer Universität. In dieser Zeit war er als Leiter

D. A. Balalykin, Russische Errungenschaften in der Physiologie der Verdauung, https://doi.org/10.1007/978-3-662-62044-1_1 
des Moskauer Militärspitals und von 1850 bis 1859 als Oberarzt des Stadtkrankenhauses tätig. Bassow führte, wie oben erwähnt, die erste Tracheotomie in Moskau durch. Nach dem Rücktritt von F. I. Inosemzew wurde er im Jahre 1859 zum Direktor der Chirurgischen Klinik der Moskauer Universität berufen, die er bis an sein Lebensende leitete.

Vor den Forschungen Bassows gab es nur wenige Arbeiten zur Magenphysiologie. Erste Studien zur exkretorischen Funktion des Magens sowie zu Eigenschaften des Magensafts hatten im 18. Jahrhundert Réaumur und Spallanzani durchgeführt. Der amerikanische Forscher Beaumont ${ }^{1}$ beschrieb 1834 den Fall des kanadischen Jägers Martin, der am Bauch verletzt wurde. Nach dieser Verletzung wurde Martin wieder gesund, jedoch bildete sich bei ihm eine Magenfistel, aus der Beaumont Magensaft entnahm und untersuchte. Seine Beobachtungen und Untersuchungsbefunde legte er in dem 1834 erschienenen Buch Experiments and observations on the gastric juice and the physiology of digestion vor. Nach Bassow kann man ,die Beobachtungen und Versuche von Beaumont in dem zufällig geöffneten Magen eines lebendigen Menschen als Beginn einer neuen Ära der Verdauungsforschung bezeichnen“.

Der von Beaumont beschriebene Fall war Ärzten und Physiologen jener Zeit gut bekannt. Seine Beobachtungen gaben somit den Anstoß zur Erforschung der Verdauungsfunktion anhand physiologischer Methoden. Es handelte sich dabei freilich um eine klinische Kasuistik, eine einzelne und zufällige Beobachtung, deren Kern eine bloße Beschreibung von Detailphänomenen darstellt. Es ist zu betonen, dass sogar die dauerhafte Beobachtung des Patienten nicht zur Idee führte, den Einzelfall als ein mögliches experimentelles Modell des Zugangs zum Magen zu sehen. In den Jahren nach der Veröffentlichung von Bassows Arbeit und ähnlichen Beobachtungen entbrannte im Ausland eine aufgeregte Diskussion über die Erstbeschreibung. Es muss darauf hingewiesen werden, dass nicht nur Beaumont, sondern auch andere Forscher, die mit dem Fall des Patienten Martin vertraut waren, aus ihm zunächst keine weiteren Schlüsse zogen.

Die Epoche der Entdeckungen von Bassow war die Zeit des aktiven Eindringens der Naturwissenschaft in die Medizin. In diesem Sinne darf man annehmen, dass die Erfindung von Bassow zum damaligen Zeitgeist passte.

Die von ihm 1842 im Rahmen eines Experiments entwickelte Magenfistel war keine bloße Analogie zu dem von Beaumont beschriebenen klinischen Fall. Die Wissenschaft jener Zeit war zu einer solchen Entwicklung bereit; sie wartete gleichsam darauf. Dies wird durch die Tatsache bestätigt, dass der norwegische Arzt Egeberg bereits 1837 den Gedanken äußerte, dass man bei Tierexperimenten eine Fistel entwickeln könne, die der Fistel des Kranken von Beaumont ähnlich sei. Egeberg versuchte aber nicht, seine Idee zu verwirklichen.

\footnotetext{
${ }^{1}$ A. I. Schtscherbakow bezieht sich auf eine Literaturquelle, nach der ein ähnlicher Fall vom österreichischen Arzt Helm 1803 beobachtet wurde.
} 
Wir legen besonderen Nachdruck auf Vorstehendes, um Folgendes zu betonen: Die Erfindung von Bassow war kein zufälliger glücklicher Einfall, sondern eine sinnvolle Antwort auf Bedürfnisse der Zeit. Die Geschichte der Wissenschaft lehrt, dass jede neue Idee nicht unvermittelt von einer einzelnen Person entwickelt wird, sondern das Ergebnis der Leistung mehrerer Forscher ist. Zum Erfinder und Ausgangspunkt einer neuen Entwicklung wird indes der Gelehrte, der ein Ergebnis als Erster erzielt - indem er den wissenschaftlichen Ertrag auf einem vorher gut bereiteten Boden einbringt.

Insgesamt ist die Geschichte der Chirurgie durch die Situation gekennzeichnet, dass operative Ideen, spontan umgesetzt, grundlegenden Untersuchungen der Organfunktionen vorangehen. Magenresektionen beispielsweise wurden von Chirurgen entwickelt und angewandt, ohne dass sie das Wesen der Erkrankungen voll erfassten. Gelehrsamkeit, Fachkenntnis und klinische Eingebung ließen sie die operativen Entscheidungen fällen. Erst später erschienen Publikationen von Physiologen, in denen die Wirkungen der Eingriffe in den Organismus beschrieben und Vor- und Nachteile solcher Interventionen erörtert werden. Es waren die Zeiten der Universalgelehrten, zu denen Bassow zählte.

Wir versuchen die Beweggründe anschaulich zu machen, die ihn zur Auseinandersetzung mit den Beobachtungen Beaumonts veranlassten. Seine ersten Versuche zur Entwicklung einer künstlichen Magenfistel nahm Bassow im September 1842 vor. Über diese berichtete er in einer Sitzung der Moskauer Gesellschaft der Naturforscher im November des gleichen Jahres.

Hunde mit einer künstlichen Magenfistel wurden von Bassow, damals Prosektor des Instituts für Physiologie der Moskauer Universität, erstmalig in den Physiologievorlesungen von Professor A. M. Filomafitski gezeigt. Darauf weist Bassow selbst in seinen Bemerkungen über den künstlichen Weg in den Magen der Tiere hin. Er schreibt, dass ,einige dieser Beobachtungen und Versuche bereits während der Vorlesungen vom Professor der Moskauer Universität Filomafitski vorgeführt wurden, der bekanntlich die Ehre der Einführung physiologischer Tierexperimente während der Vorlesungen an dieser Universität besitzt“. ${ }^{2}$

Die Ergebnisse der Tierversuche mit einer künstlichen Fistel wurden in französischer Sprache Ende 1842 in den Bulletin de la Société impériale des naturalistes de Moscou veröffentlicht. In russischer Sprache erschienen sie 1843 in den Beiträgen über Heilwissenschaften, die von der Medizinisch-Chirurgischen Akademie in Sankt Petersburg herausgegeben wurden.

Bassow beschrieb ausführlich die Methode der Operationsführung unter Beifügung entsprechender Abbildungen. Er vertrat die Auffassung, der beste Zugang zum Magen sei der Schnitt an der Vorderseite des Bauches. Der transthorakale Zugang sei schwieriger und gefährlicher. Unabdingbar sei, den Tieren 16 bis 20 Stunden vor

${ }^{2}$ Bassow, W. A., Bemerkungen über den künstlichen Weg zum Magen der Tiere. Anthologie der Geschichte der russischen Chirurgie, Bd. 2. S. 23. 
der Operation kein Futter zu geben. Der eigentliche Eingriff wird von Bassow recht detailliert und präzise beschrieben. Sein Verfahren wird bis heute als eines der besten angesehen und $\mathrm{zu}$ experimentellen Zwecken angewandt. Bassow beschrieb auch ausführlich die Pflege der Tiere in der postoperativen Phase. Die von ihm eingeführte Diät sah folgendermaßen aus: In den ersten drei Tagen erhielten sie nur Wasser und dünnen Haferschleim, ab dem vierten Tag Haferschleim mit Rindfleisch, ab dem neunten Tag gewöhnliches Hundefutter einmal täglich.

Die Nachbehandlung der Wunde erfolgte nach den damals üblichen chirurgischen Regeln. Die Heilung erfolgte per primam intentionem. Nach der Wundheilung blieb eine Öffnung im Magen und an der Bauchwand erhalten, aus der man leicht Magensaft entnehmen konnte.

In den Jahren, die auf Bassows Entdeckung folgten, befassten sich viele Forscher mit der Gastrostomie- darunter Watson in den USA, Blondlo in Frankreich, etwas später Sedillot. Dies bestätigt ein weiteres Mal die Bedeutung der Arbeit von Bassow. Von den Gelehrten, die sich mit der Problematik des operativen Zugangs zum Magen befasst hatten, war er der Erste, der hierzu ein Experiment durchführte. Andere gelangten zwar auch an das gewünschte Ziel, erreichten aber die Ziellinie mit einer gewissen Verspätung. Dies führte zu harten Auseinandersetzungen über die Frage, wer als „Vater der Gastrostomie" gelten kann. Ansprüche erhoben die oben erwähnten Watson, Blondlo und Sedillot. Sie entbehren jeder Grundlage, denn Blondlo z. B. veröffentlichte seine Versuchsergebnisse ein Jahr nach Bassow.

Der bekannte Chirurg I. Ja. Kurbatow bemerkte in seiner Doktorarbeit „Über den künstlichen Weg zum Magen“ (1879) zu den falschen Behauptungen von Blondlo und Watson: „Es lässt sich nicht lediglich durch die Unkenntnis der russischen Sprache, in der der Aufsatz von Professor Bassow erschien, seitens der ausländischen Wissenschaftler erklären. Denn er wurde 1842 auch in dem Informationsblatt der Moskauer Gesellschaft der Naturerforscher veröffentlicht und diese Informationsblätter erschienen stets, wie bekannt, in französischer Sprache. “3

Bekannte und angesehene Physiologen jener Zeit wie Schiff und Donders verteidigten die Vorrangstellung von Bassow. In seiner Dissertation führt Kurbatow eine Erklärung von Schiff zur Verteidigung der Priorität des russischen Wissenschaftlers an. Auch Pawlowwar ein leidenschaftlicher Verteidiger der Priorität von Bassow. In seiner Dissertation „Zur Lehre von den Magenoperationen“ (1883) schrieb L. Fidler: „Bassow hat die Gastrostomieoperation durchgeführt und ihre Heimat ist unser Vaterland.“

Klären wir aber die Frage nach den wissenschaftlichen Zwecken, die Bassow mit seinen Versuchen zu beantworten beabsichtigte.

Seinen Beitrag zu den durchgeführten Versuchen und den erzielten Ergebnissen beginnt er mit der Problematisierung von Beweisfähigkeit und Wahrheit einer wissen-

${ }^{3}$ Kurbatow, I. Ja., Über den künstlichen Weg zum Magen: Medizinische Doktorarbeit, Moskau, 1879. S. 5. 
schaftlichen Beobachtung. Bassow vertritt das Prinzip der Wahrheitsbeweisfähigkeit in der Wissenschaft, anknüpfend an Descartes, nach dem die Offensichtlichkeit als Wahrheitskriterium dient. „Es ist bekannt“, schreibt Bassow, „,dass die Offensichtlichkeit eine notwendige Bedingung für die Überzeugung von der Wahrheit ist und jede Wissenschaft wird erst dann zur Vollkommenheit gebracht, wenn die darin dargelegten Wahrheiten offensichtlich, sozusagen spürbar sind. Die Offensichtlichkeit ist umso notwendiger, je komplizierter die gegebene Erscheinung ist, je mehr Umstände vorhanden sind, die vom Forscher erschlossen werden müssen. Zu solchen Erscheinungen im Tierreich gehört die Magenverdauung. “4

Die Komplexität der Magenfunktionen, die Unmöglichkeit ihrer unmittelbaren Beobachtung sowie das Fehlen von mehr oder weniger genauen Erkenntnissen waren der Grund für eine große Anzahl von widersprüchlichen und sich gegenseitig ausschließenden Ansichten über das Wesen der Funktionen, die der Magen beim Verdauungsprozess ausübt. „Anzunehmen ist“, bemerkte Bassow, „dass die große Anzahl von unterschiedlichen Säugetieren eine Begründung für die Vielzahl von Theorien liefert. Es scheint jedenfalls niemand daran zu zweifeln, dass der Mangel an Beobachtungen und Versuchen hinsichtlich dessen, was während der Verdauung im Magen vor sich geht, der hauptsächliche Grund dafür war, dass gewisse Widersprüche bezüglich dieses tierischen Vorgangs entstanden sind und weiter entstehen. "5

Mit dem Magenfistel-Experiment sah Bassow eine grundsätzliche Verbesserung der Untersuchungsbedingungen gegeben. Die zufällige klinische Einzelbeobachtung war bestätigt, war nun ein gesetzmäßig auftretendes Phänomen, d. h. eine präzise wissenschaftliche Tatsache. Die Einzelbeobachtung war in kontrollierbare und unter bestimmten Bedingungen leicht reproduzierbare wissenschaftliche Daten gefasst worden. Beaumonts Fallbeschreibung hatte zu Bassows Experiment geführt, der sich fragte, ob das Anlegen einer künstlichen Öffnung im Magen der Tiere tatsächlich einen Beweis liefert: „Die acht von uns im laufenden Jahr vorgenommenen Hundeversuche entscheiden die gestellte Frage bejahend“" 6 befand er.

Hier stimmt die Gedankenfolge von Bassow erstaunlicherweise mit der Philosophie der experimentellen Medizin von Pawlow überein. Der Gedanke von Bassow, dass die Beobachtung eine passive und das Experiment eine aktive Erkenntnismethode ist, welche „die Zufälligkeit der Willkür des Naturforschers unterwirft“, steht erstaunlicherweise im Einklang mit den späteren Gedanken von Pawlow zur Rolle von Beobachtung und Experiment. „Die Beobachtung“, hielt Pawlow fest, „stellt eine für die Untersuchung einfacherer Erscheinungen hinreichende Methode dar. Je komplizierter aber die Erscheinung ist (und was ist komplizierter als das Leben?), desto eher ist der Versuch unvermeidlich. Nur der Versuch, der durch nichts außer den natürlichen Grenzen der

\footnotetext{
${ }^{4}$ Anthologie der Geschichte der russischen Chirurgie, Bd. II., S. 19-20.

${ }^{5}$ Anthologie der Geschichte der russischen Chirurgie, Bd. II., S. 20.

${ }^{6}$ Anthologie der Geschichte der russischen Chirurgie, Bd. II., S. 20.
} 
Erfindungsgabe menschlichen Geistes eingeschränkte Versuch, vollendet und krönt das Werk der Medizin [...]. Anders gesagt, sammelt die Beobachtung das, was ihr die Natur anbietet; der Versuch dagegen nimmt von der Natur das, was er will."7

Mit einer erstaunlichen Scharfsichtigkeit sagte Bassow die Bedeutung seiner Entdeckung für die Entwicklung der Magenchirurgie voraus, deren Beginn mit den Operationen zur Entwicklung einer Magenfistel zu Heilzwecken Ende der 1840er Jahre anzusetzen ist. „Außer physiologischer Anwendung“, schrieb er, „bestätigen geglückte Tierversuche - und auch Notfalleingriffe beim Menschen - die Sicherheit der absichtlichen Magenöffnung und des Magenschnitts, wie sie beispielsweise Bouchet de Jyon zur Entfernung einer verschluckten Gabel bei einer Frau vorgenommen hat. Dieselben Versuche weisen auf die Möglichkeit einer solchen künstlichen Öffnung bei einem Menschen hin, wenn der natürliche Weg für die Aufnahme und den Durchgang von Nahrung und Trank durch Geschwülste u. a. geschlossen bzw. versperrt ist. Es kann sich auch um die künstliche Öffnung bei der Heilung von Polypen handeln, die im unteren Teil des Speisereservoirs in der Magenhöhle wachsen, sowie bei anderen Krankheiten, die wegen der Unmöglichkeit eines unmittelbaren Zugangs zum Magen zu den unheilbaren zählen." 8

In der Physiologie der Verdauung, darunter der Magenverdauung, trat und tritt die Fistel vor allem als eine Methode auf, ohne die die weitere Entwicklung der Wissenschaft unmöglich gewesen wäre. In diesem Zusammenhang sind die Worte von Pawlow zu erwähnen: „Es wird häufig und nicht ohne Grund davon gesprochen, dass sich die Wissenschaft ruckartig und stoßweise bewegt, abhängig von den durch die Methodik verursachten Erfolgen. Mit jedem Schritt der Methodik vorwärts steigen wir sozusagen eine Stufe höher, von der aus sich eine freiere Aussicht auf noch nie gesehene Gegenstände bietet."

Die wissenschaftliche Vorrangstellung von Bassow lässt sich in zweierlei Hinsicht belegen, nämlich experimentell und klinisch. Er sah in der Anlage einer Magenfistel eine streng wissenschaftliche, experimentelle Methode und zugleich ein chirurgisches Heilverfahren. Im Protokoll der oben erwähnten Sitzung der Moskauer Gesellschaft für Naturforscher vom 14. November 1842 sind die eventuellen Anwendungsgebiete wie folgt skizziert: „Der Autor meint damit eine Möglichkeit für die Beobachtung der Verdauung zu haben und wird sie dereinst auch für die ärztliche Behandlung einiger Krankheiten nutzen können. “Es ist durchaus angebracht, hier von der Geburt der experimentel lenMagenchirurgie zu sprechen.

Seit dem Ende 1840er Jahre fand die Gastrostomie als eine chirurgische Therapiemethode immer häufiger Anwendung. Rund sieben Jahren nach der Mitteilung von Bassow über seine Erfindung, am 13. November 1849, legte der französische Chirurg S. Sedillot in Straßburg einem 52-jährigen Kranken, der an einem Speiseröhrenverschluss

\footnotetext{
${ }^{7}$ Anthologie der Geschichte der russischen Chirurgie, Bd. I., S. 525.

${ }^{8}$ Anthologie der Geschichte der russischen Chirurgie, Bd. II., S. 23.
} 
infolge von Krebs litt, eine Magenfistel an. Der Kranke starb einige Stunden nach der Operation. Die erste Gastrostomie in Russland wurde von W. F. Snegirew am 9. Januar 1877 vorgenommen. Über sie wurde in der Sitzung der Moskauer PhysischMedizinischen Gesellschaft am 7. März desselben Jahres berichtet. In der Folge kam es aber nicht zum erwarteten breiten Einsatz des Verfahrens in der Praxis, nicht in der klinischen und nicht in der experimentellen Chirurgie. Woran lag das?

Wir beginnen damit, dass Bassow die eingeschränkten Möglichkeiten der Anwendung seiner Magenfistel selbst erkannte: „Wir schätzen “, so notierte er, „die oben genannten Versuche nicht in jedem Fall als vollkommen ein. Allerdings zeigen sie die Möglichkeit eines künstlichen Zugangs in den Magen von Tieren, der erforderlich ist, um den Verdauungsprozess im Magen unmittelbar zu beobachten." Mit diesen Worten bekundete er wiederum seine für einen großen Experimentator charakteristische Sehergabe, wenn auch in einem für seine Entdeckung negativen Sinne. In der Tat führte die operative Gastrostomie, die mit seinem Experiment erstmals in die Tat umgesetzt worden war, zu keinen schnellen Ergebnissen - weder auf dem Feld der experimentellen noch auf dem Gebiet der klinischenMagenchirurgie.

Der langsame Fortschritt der operativen Magenchirurgie ist leicht zu erklären. Erstens stellten die Versuche klinischer Anwendung der Gastrostomie zunächst eine inadäquate Übernahme der experimentellen Methode dar. Es ergaben sich Probleme, die mit der Hautmazeration um die Wunde, dem Fehlen eines Obstipationsmechanismus und der grundsätzlichen Unregelbarkeit der Fistelfunktion im Zusammenhang standen. Zweitens hatten die Chirurgen Mitte des 19. Jahrhunderts nur eine vage Vorstellung von der normalen und der pathologischen Physiologie des Magen-Darm-Kanals. Die Möglichkeit des operativen Zugangs zum Magen war noch gestern so irreal erschienen, dass sich die Auffassung hielt, der Eingriff sei nutzlos. Da drangen die Ärzte in den Magen ein, und was nun? Wie funktioniert der Magen denn eigentlich? Wie sehen die von den Ärzten anzuwendenden physiologischen Standards aus? Großartig, man hat den abgesonderten Magensaft aufgefangen, aber wie sollte dieser Saft überhaupt beschaffen sein? Auf diese und ähnliche Fragen gab es zunächst keine Antworten.

Nachfolgend werden wir ausführlich zeigen, was in der klinischen Chirurgie Mitte des 19. Jahrhunderts genau geschah. Das Erstaunlichste waren fehlende Fortschritte bei der physiologischen Erforschung des Magens. Dies lag daran, dass fast nichts über das gesunde Organ bekannt war. Die Anlage einer Magenfistel, die eine Revolution darstellte, hielt man jedoch nicht für die angemessene Standardtherapie. Sie eröffnete zwar die Möglichkeit, Magensaft zu untersuchen. Es stellte sich aber heraus, dass diese Flüssigkeit in der Regel nur in ungenügender Menge und in nicht ausreichender Reinheit gewonnen werden konnte. Erst nach vielen Jahren, als Pawlow das Anlegen der Magenfistel experimentell durch die Methode der ,Scheinfütterung“ ergänzt hatte, wurde damit das Untersuchungsmodell der Magenfunktionen entworfen. Erstaunlicherweise fiel diese Methode von Pawlow mit der Logik der Erfindung von Bassow zusammen. Es handelte sich neuerlich um den Schritt von einer klinischen Kasuistik zu einem experimentellen Modell. 
Wie oben geschildert, wurde der Grundstein zur Entwicklung der Magenchirurgie durch die Entwicklung der operativen Gastrostomie im Hundeexperiment von Wassili Alexandrowitsch Bassow, Doktor der Medizin, habilitierter Chirurg und Professor an der Moskauer Universität, gelegt.

Der Begriff „Gastrostomie“ wurde 1846 vom französischen Chirurg S. Sedillot geprägt. Er war es, der, wie ebenfalls bereits erwähnt, als Erster einen solchen Eingriff an einem Menschen zu Heilzwecken vornahm. 1846 reichte Sedillot drei Beiträge über „Gastrostomie fistuleuse“ bei der Pariser Akademie ein. Mit seinen Aufsätzen, seinem Plan des beabsichtigten Operationsverfahrens sowie den positiven Ergebnissen von Tierversuchen wollte er die Mitglieder der Akademie vom Nutzen einer solchen Operation an Menschen überzeugen. S. S. Judin kommentiert die Beiträge von S. Sedillot gegenüber der Pariser Akademie folgendermaßen: „Er war dermaßen von seiner Idee hingerissen, dass er am Ende des ersten Beitrages ausrief: ,Das Wesen dieser Operation scheint uns so vernunftmäßig und günstig zu sein, dass wir bloß erstaunt sind, die Ersten zu sein, die sie vorgeschlagen und ihre ganze Bedeutung aufgedeckt haben."“

Freilich wusste er nicht, dass er mit all diesem um ganze vier Jahre zu spät kam, dass Bassow vor langer Zeit in Moskau Gastrostomien an Hunden erfolgreich durchgeführt und in seinen u. a. in französischer Sprache publizierten Aufsätzen klar und genau die Operationsindikationen für die Anwendung am Menschen festgelegt hatte.

Die Pariser Akademie bekundete ihr kühles Desinteresse an den Beiträgen von Sedillot.

Von der Unterschätzung der Magenfistel als einer chirurgischen Heilmethode durch die breite wissenschaftliche Öffentlichkeit zeugen weitere Fakten. Der französische Chirurg Petek de Catean fragte unabhängig von Sedillot bei der ,Société médicale de Dimai“ um Rat. Er wollte bei einem verhungernden Kranken einen solchen Eingriff durchführen, erhielt aber den Bescheid, die Gesellschaft halte „die Gastrostomie als Operation für unzulässig“.

Im selben Jahr 1846 wurde ein strenges Urteil über diesen Eingriff von dem prominenten deutschen Chirurgen Dieffenbach gefällt: „Bei solchen Operationen sind Genesungen vergeblich zu suchen, sie sind weniger von praktischer Bedeutung als Merrems sinnreicher Jugendtraum vom kranken Pförtner.“

Nach Sedillot wurde die Gastrostomie u. a. von Fenger im Jahre 1857, von Foster 1858, von Jones 1859, von Traden 1856 durchgeführt. Das von Fenger vorgeschlagene Operationsverfahren galt als das am weitesten verbreitete. Im Laufe von 29 Jahren führten europäische Chirurgen insgesamt 32 operative Gastrostomien durch. Der Ausgang aller Eingriffe war leider ausnahmslos ungünstig und erst 1875 erzielte Sydney Jones ein positives Ergebnis: Es war die 33. Magenfistelanlage beim Menschen. Die zweite erfolgreiche Gastrostomie zu Heilzwecken führte Verneuil 1876 durch. Das Leben des Patienten, an dem die erfolgreiche Operation durchgeführt wurde, stellte nach dem überstandenen Eingriff ein etwas merkwürdiges, aber drastisches Anschauungsbeispiel dafür dar, wie seltsam die Gastrostomie auf das breite Publikum jener Jahre wirkte. Der Patient wandelte die Folgen der Operation in eine Quelle seines materiellen 
Wohlstandes um: Gegen Geld zeigte er auf Reisen durch Frankreich seine Magenfistel. Das Geschäft endete mit seinem Tod nach übermäßigem Alkoholkonsum.

In Russland wurde die erste Gastrostomie an einem Patienten von W. F. Snegirew, Professor an der Moskauer Universität, durchgeführt. Über seine Operation berichtete er in der Sitzung der Physisch-Medizinischen Gesellschaft am 7. März 1877 sowie in der Zeitschrift Medizinskoje Obrazovanije (,Medizinische Ausbildung“) im gleichen Jahr. Am 9. Januar 1877 operierte er eine 30-jährige Frau. Der Eingriff dauerte drei Stunden und endete ungünstig - der Tod trat 30 Stunden später infolge eines Schocks ein.

1879 berichtete N. W. Sklifossowski in den Medizinskij westnik (,Medizinischen Mitteilungen“) über zwei von ihm durchgeführte Gastrostomien.

1883 brachte L. Fidler in seiner Habilitationsschrift die folgende Statistik der in Russland von 1877 bis 1882 durchgeführten Gastrostomien:

$\begin{array}{ll}\text { „1877 } & \text { - Snegirew - Moskau, Tod. } \\ 1879 & \text { - Sklifossowski - Sankt Petersburg, Tod. } \\ 1879 & \text { - Sklifossowski - Sankt Petersburg, operativer Erfolg, Tod am 19. Tag. } \\ 1880 & \text { - Jazenko, Kiew, Genesung. Ein Jahr später bescheinigt. } \\ 1880 & \text { - Kolomnin, Sankt Petersburg, Tod. } \\ 1881 & \text { - A. Kni, Moskau, Tod. } \\ 1881 & \text { - P. Pelechin, Sankt Petersburg, operativer Erfolg, Tod in der 3. Woche. } \\ 1881 & \text { - Stukowenkow, Moskau, Tod am 6. Tag. } \\ 1882 & \text { - A. Kni, Moskau, operativer Erfolg. } \\ 1882 & \text { - A. Kni, Moskau, operativer Erfolg. } \\ 1882 & \text { - M. Kitaewski, Sankt Petersburg, operativer Erfolg. } \\ 1882 & \text { - W. Dianin, Sankt Petersburg, Tod.“ }\end{array}$

L. Fidler teilt drei weitere 1882 durchgeführte Gastrostomien mit, ohne Autoren anzugeben. Ende 1882 zählte L. Fidler alles in allem 15 in Russland binnen fünf Jahren durchgeführte Gastrostomien.

Den oben angeführten Angaben ist zu entnehmen, dass die postoperative Sterblichkeit bei Gastrostomien damals sehr hoch war, denn in den zwölf von L. Fidler erwähnten Fällen kam es nur in einem zur Genesung.

1885 konnte E. Albert mit der weltweit größten Erfahrung auf dem Gebiet der operativen Gastrostomie prahlen (zwölf Eingriffe). Von den russischen Chirurgen führte A. D. Kni zu jener Zeit die größte Anzahl von Gastrostomien - neun Operationen - in Moskau durch. N. A. Weljaminow und N. W. Sklifossowski führten je sechs Gastrostomien durch, I. F. Sabaneew fünf Operationen. Letzterer entwickelte eine Modifikation, um eine Verzögerung der Passage zu erreichen, bei der die Muskeln der vorderen Bauchwand eine wichtige Funktion übernahmen. Die Ideen von Sabaneew hatten große Bedeutung für den Einsatz der Gastrostomie in der klinischen Praxis. Unten gehen wir auf seine Neuerungen in der operativen Magenchirurgie näher ein.

Die russische Chirurgie sammelte eine beträchtliche Erfahrung. Zugleich ist $\mathrm{zu}$ bemerken, dass in der Klinik von Billroth in Wien von 1880 bis 1884 nur vier 
Gastrostomien durchgeführt wurden. 1885 führte Zesas in einer weltweiten Statistik 129 Gastrostomien auf.

Wenn man den Berechnungen von Kni traut, so lag Anfang 1886 die weltweite Erfahrung, einschließlich russischer Chirurgen, bei 169 Gastrostomien.

Äußerst widersprüchlich war die Einschätzung der Operationsergebnisse. Nach Ausweis verschiedener Autoren schwankte die Sterblichkeit zwischen $75 \%$ und 27,5\%. Laut Schätzungen von Kni wurden nach Stand des Jahres 1886 nur 56 von den 169 Patienten wieder gesund. Die allgemeine Einschätzung der Effizienz des Verfahrens wurde erstens durch geringe Eingriffserfahrungen und zweitens dadurch erschwert, dass ein Teil der Interventionen in vorantiseptischer Zeit durchgeführt wurde und die Gründe für letale Ausgänge demzufolge kaum objektiv zu beurteilen sind. Es liegt aber auf der Hand, dass auch der beste chirurgische Eingriff durch eine hohe Letalität infolge postoperativer Infektionen diskreditiert werden kann. Deswegen lassen sich die Resultate der in vorantiseptischer Zeit durchgeführten Operationen nicht aus dem Blickwinkel einer Effizienzbeurteilung der Methode an sich betrachten.

Vitringa analysierte Ergebnisse einer Gastrostomie vor und nach dem Einsatz von antiseptischen Methoden in der klinischen Praxis. Nach seinen Schätzungen gab es in der vorantiseptischen Zeit nur $3 \%$ Genesungen. Nachdem Chirurgen mit der Anwendung von antiseptischen Verfahren begonnen hatten, erhöhte sich die Genesungsquote auf $47 \%$. Nach Angaben von Kni erreichte die Sterblichkeit infolge der Gastrostomie in der vorantiseptischen Zeit $55 \%$; nach dem Einsatz antiseptischer Methoden im Jahr 1886 sank die Sterblichkeit auf $27 \%$.

Sehr widersprüchlich sehen auch die Angaben verschiedener Autoren zur Überlebensdauer der Kranken nach einer Gastrostomie aus. Nach Schätzungen von Kni betrug die durchschnittliche Lebensdauer 20 Tage, nach Angaben von Petit 14 Tage. Anhand der Auswertung von 167 Operationen gibt Grass die durchschnittliche Überlebensdauer mit 33 Tagen an.

Viele Autoren weisen darauf hin, dass die Sterblichkeit infolge des Eingriffs neben allen sonstigen Gründen von der richtigen Bestimmung der Operationsindikation abhing. Billroth z. B. maß dieser Tatsache allergrößte Bedeutung bei. Die meisten Gastrostomien jener Zeit wurden im Zusammenhang mit einem Speiseröhrenverschluss infolge eines Malignoms des Ösophagus bzw. der Kardia oder wegen einer narbigen Speiseröhrenstenose durchgeführt.

In all diesen Fällen wurde die Gastrostomie als eine ursprünglich palliative Operation vorgenommen. Letztendlich hing das Schicksal des Kranken somit nicht von dem Eingriff, sondern von der Entwicklung der Grunderkrankung ab. Eine Ausnahme von dieser Regel stellten die Fälle der narbigen Speiseröhrenstenose verschiedener Genese dar, sofern die Magenfistel die Ernährung des Kranken und damit sein längerfristiges Überleben sicherstellte.

Um die Mitte der 1880er Jahre trat offen zutage, dass die wesentlichen Ursachen der hohen Sterblichkeit bei Gastrostomien in der unzureichenden Erfahrung der Chirurgen 
lagen - einerseits infolge einer geringen Zahl von Eingriffen, andererseits aufgrund einer unzureichenden Bestimmung der Operationsindikationen.

Nach dem Einsatz von antiseptischen Methoden sowie der Antiseptikund Aseptikin der Praxis wurde die Kachexie zur Hauptursache der Sterblichkeit nach Gastrostomie, weil es nicht gelang, ein normales Ernährungsregime über die Fistel zu erreichen. Die durch die Fistel eingeführten harten Speisen wurden häufig reflektorisch aus dem Magen ausgeschieden und dieser Nahrungsverlust wurde zu einer regelrechten Geißel der Kranken und der Ärzte. Die Chirurgen strebten danach, das Operationsverfahren zwecks Beseitigung dieser drohenden Gefahr zu verbessern. Bis Anfang der 1880er Jahre war die Fenger-Methode besonders beliebt, bei der die Fistel mit einem speziellen Obturator mechanisch verschlossen wurde, was eine Reihe von Unannehmlichkeiten für den Kranken schuf und schlechte Ergebnisse nach sich zog.

Es kam schließlich die Zeit, als man an die Ausführung einer Gastrostomie zwei von Sabaneew formulierte Hauptforderungen stellte: nämlich erstens diese Operation quoad vitam möglichst risikoarm zu gestalten und zweitens eine möglichst zweckmäßige Funktion der angelegten Magenfistel zu gewährleisten.

Zur Beseitigung negativer Folgen der Gastrostomie wurden mehrere Verfahren vorgeschlagen, die sich - nach operativen Grundsätzen sowie Anwendungslogik - in drei Gruppen einteilen lassen. Erste Gruppe: Entwicklung einer aufrechten Lippenfistel, durch die man in den Magen nicht nur flüssige, sondern auch harte Speisen in einer Menge einführen kann, die für eine normale Ernährung des Kranken ausreicht (Trendelenburg u. a.). Zweite Gruppe: Verfahren zur Anlegung eines aus der Magenwand gebildeten schrägen Kanals (Witzei, Marwadel, Schnizler). Zur dritten Gruppe gehörten die Verfahren zur Bildung einer „Quetsche“ aus Bauchmuskeln oder Bauchwandfalten (Hacker, Ullmami, Kader). Durch Unterbringung der Magenfistel in der Muskelmasse versuchte Hacker beispielsweise einen natürlichen Wulst für die Fistel aus den sich kontrahierenden Fasern zu bilden. Hahn brachte die Magenfistel im achten Zwischenrippenraum unter, was ihm harte Wände sicherte und einen dichten Verschluss der Fistel nach der Zuführung von Speisen ermöglichte. Die von Hahn mitgeteilten Ergebnisse waren am aussichtsreichsten.

Sabaneew sah die Nachteile aller Varianten darin, dass ,die nach einem der klassischen Verfahren entwickelte Magenfistel ihren anatomischen Eigenschaften nach dem Typ der beschwerlichsten Darmfisteln angehört, nämlich dem Typ der Lippenfisteln, der unter gewissen Umständen das Leben des Kranken gefährdet. Die periodische Kontraktion des Pylorus, die in den Magenfisteln die Funktion eines Sporns ausübt, hat samt der Peristaltik des Magens zur Folge, dass sein Inhalt fast vollständig bereits während des Verdauungsaktes durch den kurzen Kanal der Fistel (die sich außerdem manchmal übermäßig ausdehnt, besonders unter Einfluss von Obturatoren) ausgeschieden wird, was sich selbstverständlich äußerst unangenehm auf die Ernährung des Kranken auswirkt"“.

Zur Beseitigung der geschilderten Nachteile äußerte Sabaneew 1889 die Idee, dass „die operative Gastrostomie nur dann die in sie gesetzten Hoffnungen rechtfertigen wird, 
wenn ein Verfahren entwickelt wird, das die dadurch entstehende Magenfistel dem Typ der Lippenfisteln annähert“.

Als Resultat dieser Idee erwies sich das von ihm ursprünglich an Leichen entwickelte Verfahren für die Anlage einer Magenfistel, das sich dem Typ der Darmlippenfistel annähert. Dabei liegt eine der Wände auf einer harten Knochenunterlage.

Da wir in der medizinhistorischen Literatur keine Analyse des Beitrags des hervorragenden russischen Chirurgen I. F. Sabaneew, der in die Schatzkammer der Magenchirurgie gehört, finden, halten wir es für zweckmäßig, die Beschreibung des von ihm entwickelten Verfahrens zur Anlage einer Magenfistel so wiederzugeben, wie sie der Autor selbst verfasste. Hierzu erlauben wir uns, das nachfolgende lange Zitat anzuführen.

„Die Durchführung dieser Operation“, so Sabaneew, „,besteht aus den vier folgenden Schritten.

Der erste Schritt - die Bauchdeckeneröffnung: Der Operateur, indem er sich links von dem Kranken einrichtet, dessen linker Arm etwas seitwärts abgewinkelt ist, macht einen Hautschnitt 7-8 cm lang am Rand der linken falschen (asternalen) Rippen, beginnend auf der Höhe der Knorpelverbindung der 8. und 9. Rippe. Nach Aufschneiden der Haut, des Unterhautgewebes, der Faszie und der zwei Muskelschichten ebenso wie des abdominalen Fettgewebes öffnet man - während man die verletzten Gefäße abbindet - zwischen zwei Federzangen das Bauchfell und fixiert seine beiden Ränder durch Ligaturen.

Der zweite Schritt - Auffindung des Magens: Die Wundränder auseinanderbringend, findet der Operateur den Magen, indem er zur Bestimmung seine Lage zwischen dem kleinen und dem großen Netz benutzt, wobei das erste durch seine Lage gegenüber der unteren Leberoberfläche bestimmt wird. Nachdem der Operateur den Magen aufgefunden hat, zieht er ihn in die Bauchöffnung und versucht, einen Teil seiner Vorderwand im Bereich des Fundus ventriculi in eine Falte zu ziehen.

Das dritte Schritt - Einnähen des Magens: Nach dem vorsichtigen Ausziehen der oben genannten Magenfalte durch die Bauchwunde legt der Operateur sie unter den Rand der linken Rippen. Dann, unter Fortsetzung vorsichtigen Ziehens des Magens in seiner neuen Lage, bestimmt der Operateur den Punkt unter den Rippen, den man ohne besonderen Zug mit der Spitze der ausgezogenen Magenfalte erreichen kann, wenn man sie nach oben und außen mobilisiert. Normalerweise befindet sich dieser Punkt auf der Höhe der sechsten Rippe bzw. gleich darunter, mit einem 1-2 cm langen lateralen Abstand zur linken Mamillarlinie. Nach der Kennzeichnung dieses gesuchten Punkts spannt der Operateur die Spitze der Magenfalte in eine Darmklemme und übergibt diese in die Hände des Assistenten. Danach macht der Operateur einen 3-4 cm langen Schnitt durch die Weichteile bis zu den darunterliegenden Rippen parallel zum Rippenrand auf der Höhe des Punktes, bis zu dem sich der Magen ziehen ließ. Durch den Schnitt werden nun alle Weichteile mit einer stumpfen Kornzange von den darunterliegenden Knorpeln aus von kranial nach kaudal und von lateral nach medial in der Richtung des ersten Schnittes getrennt, sodass eine durch die genannten Schnitte begrenzte Brücke 
aus Weichteilen gebildet wird, wobei zwischen dieser Brücke und den darunterliegenden Rippen ein Kanal entsteht, dessen breite Basis in den ersten der Schnitte und die enge Spitze in den zweiten der Schnitte führt.

Nachdem der Operateur durch den auf diese Weise entstehenden Kanal die Kornzange gezogen hat, fasst er damit die Spitze der vom Assistenten gehaltenen Magenfalte und führt sie so unter die Brücke, dass ihre Spitze $1 \mathrm{~cm}$ hoch aus dem äußeren Schnitt vorspringt; dann übergibt er die Kornzange dem Assistenten, der den Magen in dieser Lage festhält. Danach verbindet der Operateur mit möglichst dicht anzulegenden Nähten den inneren Schnittrand des Peritoneum parietale mit dem Peritoneum viscerale, der Basis der ausgezogenen und in der oben ausgeführten Lage festgehaltenen Magenfalte. Nach dem Anlegen dieser Nähte führt der Operateur die Magenfalte unter der Hautbrücke zurück und verbindet den äußeren Rand des Schnitts des Peritoneum parietale mit dem Peritoneum viscerale der daran angrenzenden Magenseite, während der Assistent den einzunähenden Magenteil nach innen zurückzieht. Diese Naht lässt sich ohne vorherige Mobilisierung der einzunähenden Magenfalte unter die Hautbrücke vornehmen. Aber bei Einhaltung des bezeichneten Verfahrens gelingt es, wovon mich Übungen an Leichen überzeugten, die zusammenzunähenden serösen Überzüge genauer zu verbinden. Wenn nach der Anlegung dieser Nähte, an der ganzen Kreislinie der ausgezogenen Magenfalte entlang, in den Schnittwinkeln insbesondere des unteren Peritoneums der Bauchwand kleine Öffnungen bleiben, werden sie durch Nähte verschlossen. Die mit ihrer Basis eingenähte Magenfalte ist wieder unter die Hautbrücke zurückzuführen und an die Ränder des zweiten Schnitts mit vier Nähten anzusetzen, die folgendermaßen angelegt werden müssen: Zuerst durchsticht man die Haut und alle Weichteile des Schnittrandes, dann fasst man das Peritoneum an der aus dem Schnitt hervorstehenden Spitze der Magenfalte und danach wieder die Weichteile und die Haut, aber nun von innen nach außen, wonach die Fäden unter der Haut vernäht werden. Diese Nähte befestigen eigentlich die Magenfalte.

Das vierte Schritt: Nun öffnet man den Magen mit einem kleinen Schnitt (1-2 cm lang) an der Spitze der herausgezogenen Magenfalte und verbindet die Schleimhaut mit der Haut. Nach der Anlegung dieser letzten Reihe von Nähten wird ein Drainagerohr in die Magenhöhle durch das Loch eingeführt. Nachdem man sich vergewissert hat, dass das Drainagerohr in den Magen eingedrungen ist, fügt man die Ränder des noch nicht geschlossenen Schnitts beim Tasten der Vorderwand der eingenähten Magenfalte nach allgemeinen Regeln mit Nähten zusammen. Das eingeführte Drainagerohr wird mit einer Klemme geschlossen; dann wird ein leichter Druckverband im Bereich des Epigastriums angelegt.

Das Wesen der Operation besteht somit darin, dass die in Gestalt eines Divertikels geformte Magenfalte unter die Brücke der über dem Rippenrand liegenden Haut verlegt wird, wodurch ein von den Magenwänden belegter Gang gebildet wird, der durch die Fistel eröffnet wird, die nach außen 5-7 cm vom Rippenrand entfernt liegt. Die Wände dieses Ganges sind durch den Druck auf die darunter liegenden Rippenknorpel leicht zur gegenseitigen Berührung bis zum vollen Lumenverschluss zu bringen.“ 
In diesem Aufsatz berichtet Sabaneew von fünf Gastrostomien, die er im Zeitraum von 1890 bis 1892 nach der beschriebenen Methodik mit günstigeren Ausgängen als bei den in der damaligen Literatur wiedergegebenen Fällen durchführte.

In seinem im Jahre 1894 veröffentlichten Beitrag berichtete W. W. Lesin über günstige Ergebnisse der Fistelanlage nach diesem Verfahren. Im Aufsatz „Vier Gastrostomiefälle nach dem Verfahren von Sabaneew" wies auch G. D. Romm 1895 auf den guten Ausgang bei der Anwendung hin.

Nach der uns zugänglichen Literatur kommen wir zu der Schlussfolgerung, dass das Verfahren für die Magenfistelanlage von Sabaneew den Vorrang der russischen Chirurgie belegt. Dieser Vorrang wird von uns besonders nachdrücklich hervorgehoben, weil noch andere Chirurgen ohne allen Anlass ihre Ansprüche darauf geltend machten. Auf diesen Umstand wurde auch der eigentliche Urheber des neuen Verfahrens aufmerksam. „Am Schluss meiner Mitteilung“, schrieb Sabaneew, ,halte ich es für notwendig darauf hinzuweisen, dass Frank im Herbst vorigen Jahres eine Gastrostomie nach demselben Verfahren erfolgreich durchführte und dieses als sein eigenes Verfahren veröffentlichte. Ich aber führte diese Operation erstmalig im Mai 1890 durch und eine vorläufige Benachrichtigung über dieses Verfahren erfolgte am 15. September desselben Jahres in der Sitzung der Gesellschaft der Ärzte von Odessa (siehe die Zeitschrift Wratsch („Arzt“"), 1890, Nr. 39, S. 897).“

1898 entwickelte M. M. Trofimow eine Variante des Verfahrens zum Anlegen einer Fistel nach Kader, die er „Klappenverfahren der Magenfistelanlage“ nannte.

Dennoch bildete sich selbst gegen Ende des 19. Jahrhunderts keine klinische Tradition bzw. Schule auf dem Gebiet der Gastrostomie heraus, die zu einer verbreiteten Anwendungspraxis geführt hätte. Wie die oben angeführten Angaben belegen, verfügte die überwiegende Mehrheit der Chirurgen nicht über eine ausreichende operative Erfahrung. Wie N. A. Weljaminow hervorhebt, ,führte die Mehrheit der Operateure diesen Eingriff nur ein paar Mal durch, und eine Menge Chirurgen - nur einmal. Es ist deswegen absolut schlüssig, dass vom Standpunkt der Operationstechnik aus bei einer Reihe von Gastrostomien mehrere Fehlgriffe geschehen sind“.

Ungeachtet dessen spielte die Gastrostomie eine wichtige Rolle sowohl als ein selbständiges Verfahren als auch als eine Art Generalprobe für die „große Magenchirurgie“ bzw. Magenresektion.

Im April 1877 sprach T. Billroth auf dem VI. Kongress der deutschen Chirurgen, bei dem die Probleme der Gastrostomie und der Magenresektion diskutiert wurden, die folgenden prophetischen Worte: „Bei alledem glaube ich, dass die Magenresektion ihre Zukunft hat, aus dem einfachen Grunde, dass, wenn wir eine Fistel risikolos anlegen können, das Magenkarzinom tasten können und dabei noch etwas über seine Verbreitung sagen können, was bleibt dann unmöglich?“ Auf diese Aussage von Billroth bezogen sich J. W. Sumarokow, L. Fidler, K. F. Klein, S. S. Judin und andere Chirurgen.

In den letzten Jahren des von uns beschriebenen Zeitraums kommt es weltweit zu wichtigen Innovationen auf dem Gebiet der operativen Magenchirurgie. Es werden Grundsätze der Magenresektion und der Anastomosen-Anlegung entwickelt. Diese 
sind vor allem mit den Werken von Péan (1879), Billroth (1881) und seinen Assistenten Riediger (1881) und Belfleur (1881) sowie Richter (1881) und Kocher (1883) verbunden. Fortschrittliche Methoden der Magenchirurgie wurden in Russland einige Jahre später eingeführt. 1881 beschränkte sich die Erfahrung in operativer Magenchirurgie russischer Chirurgen auf die Gastrostomie.

Der von uns behandelnde Zeitraum in der Geschichte der Magenchirurgie war die Zeit ihrer Entstehung und ihrer ersten Schritte, die im Zeichen von enormer Sterblichkeit, sehr kurzem postoperativem Überleben der Patienten sowie der Unvorhersehbarkeit der Ergebnisse selbst unter günstigen Bedingungen standen. Deswegen muss man sich bei der Lektüre der Werke von bahnbrechenden russischen Ärzten der Magenchirurgie den Worten von Judin anschließen, der schrieb: „Wenn man ihre eigenen Aufzeichnungen im Originaltext liest, fühlt man lebhaft, wie viele Überlegungen sie anstellten, wie viele tiefe Emotionen sie bewegten auf der Suche nach den neuen Pfaden, auf denen ihnen das Glück zunächst so selten lächelte.“

Die Gastrostomie ist nicht nur der Anfang der Magenchirurgie. Diese Operation nimmt ihre Stellung im Arsenal der gesamten modernen Chirurgie ein. Wie B. W. Petrowski mit voller Berechtigung bemerkt, ,verlor diese Operation bis heute nicht an Bedeutung, denn die Magenfistel bleibt ein vortreffliches Verfahren bei einer dringlichen Operation beim Speiseröhrenverschluss und wird als eine der Stufen des Speiseröhrenplastik-Systems sowie bei inoperablen Tumoren dieses Organs benutzt“.

Open Access Dieses Kapitel wird unter der Creative Commons Namensnennung 4.0 International Lizenz (http://creativecommons.org/licenses/by/4.0/deed.de) veröffentlicht, welche die Nutzung, Vervielfältigung, Bearbeitung, Verbreitung und Wiedergabe in jeglichem Medium und Format erlaubt, sofern Sie den/die ursprünglichen Autor(en) und die Quelle ordnungsgemäß nennen, einen Link zur Creative Commons Lizenz beifügen und angeben, ob Änderungen vorgenommen wurden.

Die in diesem Kapitel enthaltenen Bilder und sonstiges Drittmaterial unterliegen ebenfalls der genannten Creative Commons Lizenz, sofern sich aus der Abbildungslegende nichts anderes ergibt. Sofern das betreffende Material nicht unter der genannten Creative Commons Lizenz steht und die betreffende Handlung nicht nach gesetzlichen Vorschriften erlaubt ist, ist für die oben aufgeführten Weiterverwendungen des Materials die Einwilligung des jeweiligen Rechteinhabers einzuholen.

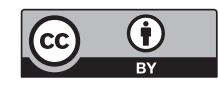

